\title{
Making resuscitation trolleys safer and more effective at an acute NHS Trust in Essex, UK
}

Matthew Ibrahim -Lead Resuscitation Practitioner-The Princess Alexandra Hospital NHS Trust

Phillip Chandler -Resuscitation Practitioner-The Princess Alexandra Hospital NHS Trust

\section{$\underline{\text { Aim }}$}

It is well accepted that the safe provision of emergency equipment in clinical areas is a must and is recommended by various groups (NICE 2010; Resuscitation Council (UK) (2015). A deep dive resuscitation trolley audit was conducted at The Princess Alexandra Hospital NHS Trust which found that only $38 \%$ of trolleys had all contents present and in date, in line with Trust policy. The aim of this project was to introduce a safe, user friendly way of ensuring emergency equipment was present.

\section{Method}

The design of the resuscitation trolleys were changed Trust wide to accommodate pre-packed sealed "Airway" and "Access" trays. These trays were packed and checked by a member of the Trusts Resuscitation Service. Items in the tray were based on national guidance and local preference. Subsequent monthly deep dive audits were carried out looking at compliance.

\section{Results}

Within four weeks of introduction of the new system compliance increased from $38 \%$ to $74 \%$ and within three months reached $100 \%$. This standard has been maintained for the past six months. A time in motion exercise was conducted which demonstrated a reduction in staff time taken to check the trolley from 34 minutes to 7 minutes. Due to the reduction in unnecessary and duplicated items, there was a cost saving of $£ 97.00$ per trolley. There was also a significant reduction in incident reporting relating to emergency equipment provision.

\section{Conclusion}

The introduction of the new trolley system has not only ensured improved safe and timely access to emergency equipment, it has yielded time and financial savings for the Trust. It is estimated that $£ 4365.00$ of savings on consumables have been realised, with Time saved on checking the trolley reinvested into patient facing activities Staff report that access to equipment during events is now much easier and safer.
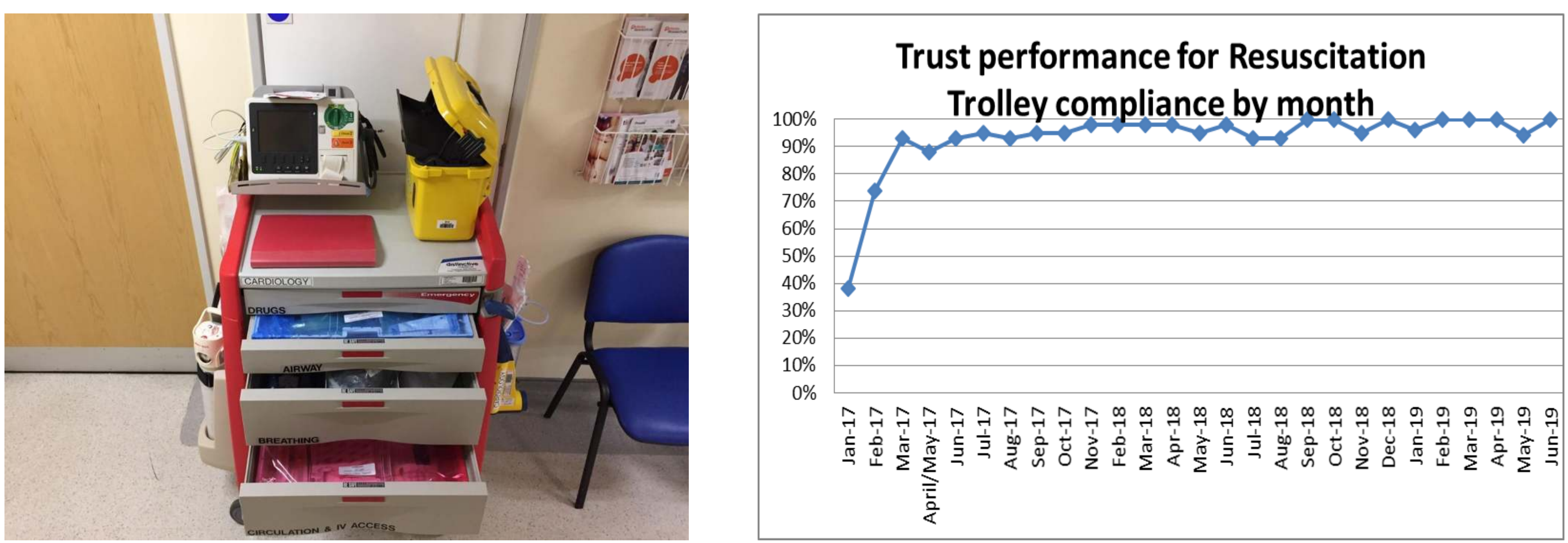